\title{
Factores de riesgo y de protección y victimización por ciberacoso entre menores en Jerez de la Frontera: un estudio exploratorio
}

Risk and protective factors, and cyberbullying victimization among minors in Jerez de la Frontera: an exploratory study

Ana María Ramírez Ganfornina

Universidad de Cádiz, Jerez de la Frontera, España

Email de correspondencia: anamariarganfornina@gmail.com

\begin{abstract}
Resumen
Los menores de edad se encuentran en una situación delicada frente a Internet, ya que están expuestos a numerosos riesgos que los hacen más vulnerables a sufrir victimización por ciberacoso. En esta investigación se realizó una encuesta a una muestra de 137 estudiantes de un instituto en Jerez de la Frontera con el objetivo de analizar la probabilidad que tienen estos menores a sufrir victimización por ciberacoso, asi como comprobar si esta depende de las conductas de riesgos y los factores de protección. Los resultados permiten concluir que los menores están expuesto a una gran variedad de conductas de riesgos, pero las que incrementan la probabilidad de la victimización por ciberacoso son las horas que pasan conectados a Internet, el numero de redes sociales en las que tienen un perfily los riesgos altos y bajos. Por su parte, los factores de protección analizados muestran efectivamente un efecto protector frente a la victimización por ciberacoso. Las implicaciones para la literatura y la prevención del ciberacoso de estos resultados son discutidas.
\end{abstract}

\section{Palabras Clave}

Encuesta, escolares, Internet, cibercrimen, factores de riesgo, estudio cuantitativo.

\begin{abstract}
Children are in a delicate situation when it comes to the Internet, as they are exposed to many risks that make them more vulnerable to cyber-bullying victimization. In this research, a survey was carried out on a sample of 137 students from a high school in Jerez de la Frontera with the aim of analysing the likelihood of these minors to suffer cyberbullying, as well as checking whether this depends on risk behaviours and protective factors. The results show that children are exposed to a wide range of risk behaviours, but those that increase the probability of cyber-bullying victimization are the hours they spend online, the number of social networks in which they have a profile and the high and low risk.s. In turn, the protective factors analysed do show a protective effect against cyberbullying. The implications for the literature and the prevention of cyberbullying of these findings are discussed.
\end{abstract}

\section{Keywords}

Survey, schoolchildren, Internet, cybercrime, risk factors, quantitative study. 
Ramírez, A. M. (2020). Factores de riesgo y de protección y victimización por ciberacoso entre menores en Jerez de la Frontera: un estudio exploratorio. Behavior \& Law Journal, 6(1), 51-63.

\section{INTRODUCCIÓN}

El uso de Internet por parte de los menores de edad conlleva un elevado número de riesgos que, a menudo, son desconocidos por los padres y madres. En general, el tipo de actividad más realizada por los españoles es el uso de la mensajería instantánea (p.ej., WhatsApp), es decir, que la mayoría de los menores de 13 a 16 años están en contacto virtual con alguien -conocido o desconocido- a diario (Garmendia Larrañaga et al., 2016). Según el Instituto Nacional de Estadística (en adelante, INE), (2018), casi la totalidad de los menores de edad españoles son usuarios de Internet y es este grupo el que más confiado se muestra en las redes y el que menor percepción de riesgo tiene. Por ello, es el grupo más vulnerable a sufrir conductas de ciberacoso.

En este estudio se analiza la relación entre la victimización por ciberacoso y la presencia de factores de riesgo en Internet o de factores de protección. En primer lugar, se exponen los antecedentes de esta investigación. A continuación, el procedimiento para la implementación de la encuesta en Jerez de la Frontera se explica en la metodología, junto a las consideraciones éticas de la investigación. Los resultados están organizados para responder a cada uno de los objetivos de la investigación. Por último, los resultados son discutidos y se proponen futuras intervenciones con los menores en Institutos de Cádiz.

\section{ANTECEDENTES DEL ESTUDIO}

\section{A. Perfil de los menores víctimas de ciberacoso}

Uno de los perfiles que más probabilidad tiene de ser víctima de ciberacoso tiene la característica de adicción a Internet, que es uno de los riesgos que puede conllevar su mal uso o uso excesivo. Así lo afirman Mishna et al. (2012) para quienes todos los estudiantes involucrados en delitos cometidos en Internet lo usan durante más de 1 hora al día. Otra característica, según Cruzado Díaz et al. (2012), es que el sexo predominante es el masculino, debido a que suelen usar aplicaciones de Internet de carácter más impulsivo. Sin embargo, Navarro y Jasinski (2013) afirman que las niñas corren mayor riesgo de experimentar ciberbullying, aunque este resultado no es consistente en la literatura sobre ciberacoso (Hinduja y Patchin, 2008). En cuanto a la edad, se sitúan entre los 13 y los 28 años, siendo los menores de 21 años los que más riesgos tienen debido a que los adolescentes son más susceptibles a desarrollar problemas adictivos (Cruzado Díaz et al., 2012). Aunque, generalmente, se ha descrito que el uso problemático de Internet lo podemos encontrar en cualquier edad, grupo social, cultural o económico (García Oliva, 2017).

Además, tenemos que tener en cuenta que nos encontramos con personas aún "inmaduras" ya que, como afirmó Oliva Delgado (2012), de acuerdo con la maduración cerebral, los primeros años de la adolescencia (sobre todo cuando la pubertad se adelanta) es una etapa de alta vulnerabilidad en la que la inmadurez de los mecanismos autoreguladores requiere una mayor supervisión por parte de los padres. A esta característica le sumamos el desconocimiento de los demás riesgos que pueden tener y la posibilidad que tienen de ser víctimas. Sin embargo, se ha estudiado que los menores con mayor percepción de riesgo en Internet tienen más habilidades para protegerse frente a los peligros online (Ramos-Soler et al., 2018).

En una investigación reciente sobre perfiles de víctimas de ciberacoso repetido en España, donde utilizaron una nueva metodología encontraron perfiles asociados a una mayor probabilidad de ser víctimas de ciberacoso (Moneva et al., 2020). En concreto, encontraron que los perfiles de menores más probables de sufrir ciberacoso eran las mujeres de entre 15 y 17 años, que pasaban entre 4 y 7 horas conectadas a Internet, tenían perfil de Snapchat, de Instagram, subían fotos a sus perfiles, y no tenían ajustes de privacidad en sus cuentas. Este perfil obtuvo una probabilidad de ser víctima de ciberacoso del $82 \%$. Por el contrario, el perfil con menor probabilidad de ser víctima de ciberacoso $(0 \%)$ es el niño de entre 12 y 14 años, que pasa entre 4 y 7 horas conectado, tiene perfiles en Instagram, Facebook, y Twitter pero estos son privados y además no sube fotos.

Podemos concluir que los resultados de los estudios sobre los perfiles específicos de menores que sufren victimización por ciberacoso no son concluyentes. Parece que hay menores de edad que normalizan los factores de riesgo a los que están expuestos, como es el número de horas que están conectados a Internet, las actividades que realizan, que lleguen a la adicción, con el tipo de personas que hablan, etc., y, si a todo esto, se le suma la poca concienciación e información que tienen sobre el uso de Internet (factores de protección) se incrementa la probabilidad de que los menores sufran victimización por ciberacoso.

\section{B. Factores de riesgo y victimización por ciberacoso}

Aunque existen muchos tipos de ciberacoso, el robo y difusión de videos y fotografías es el considerado más problemático debido a que perjudica la imagen pública del propio menor de edad. Esto suele suceder con frecuencia 
Ramírez, A. M. (2020). Factores de riesgo y de protección y victimización por ciberacoso entre menores en Jerez de la Frontera: un estudio exploratorio. Behavior \& Law Journal, 6(1), 51-63.

y se hace llamar sexting (Jiménez Iglesias \& Garmendia Larrañaga, 2015). Varios autores también encuentran en sus estudios un amplio porcentaje de victimización por difusión de imágenes desagradables, seguido de mensajes desagradables (p.ej., Gamito et al., 2017).

Además, el estatus socioeconómico alto está relacionado con el acceso a Internet en menores de edades más tempranas. El uso diario o casi diario de Internet se da en un 63\% de los hogares de estatus alto frente al 55\% de los hogares de estatus socioeconómico bajo (Garmendia et al., 2012). Garmendia (2012) también estudió otra diferencia en cuanto al contacto con extraños, mayor en el caso de que la familia del menor tenga un estatus socioeconómico alto (27\%) que bajo (19\%). Los adolescentes con disfuncionalidad familiar, provenientes de familias pequeñas, del estrato socioeconómico medio y los que utilizan más redes sociales, tienen más riesgo de presentar adicción a Internet (Aponte Rueda et al., 2017). Por otro lado, los adolescentes que viven en hogares con menores ingresos se enfrentan a más riesgos. Sin embargo, la incidencia del estatus socioeconómico sobre los riesgos online de los menores ha sido escasamente analizada (Catalina García et al., 2014). En conclusión, podemos decir que el grupo social medio sufre una mayor victimización que el grupo social alto y, sin embargo, este último tienen más tendencia a llevar a cabo conductas de riesgo.

Generalmente, los riesgos que generan mayor preocupación son los que tienen una naturaleza social. Atendiendo a esta característica mencionada, según Miranda de Larra (2005), se ha optado por dividir los riesgos en tres categorías: (1) el riesgo relacionados con la navegación mediante páginas web (2) el riesgo procedente de la participación en servicios interactivos y (3) los riesgos derivados del exceso de tiempo de exposición. En este sentido, se ha señalado que los menores que pasan mucho tiempo conectados a Internet tienen mas probabilidad de sufrir victimización por ciberacoso, y lo mismo afirman cuando habla del factor de riesgo "conversar con alguien que no conocen a través de este medio", es decir, que ambos riesgos tienen una relación positiva con la victimización por ciberacoso (Marcum et al., 2010). Además, Hinduja y Patchin (2008) encontraron que el ser víctima de acoso tradicional está relacionado con la victimización por ciberacoso.

\section{Figuras parentales y medidas preventivas}

Para entender el papel de los padres y madres en el ciberacoso es necesario conocer el significado de mediación parental, que se define, en el ámbito tecnológico, como el proceso por el que los padres y madres educan a sus hijos en el uso responsable y seguro de las nuevas tecnologías con el objetivo de protegerlos de los riesgos a los que pueden estar expuestos (Jiménez Iglesias \& Garmendia Larrañaga, 2015). La supervisión que realizan los padres y madres es muy relevante. Así lo considera el Grupo de Informática de la Sociedad de Pediatría (2007), que se refleja la importancia del control parental unida a la supervisión adecuada ya que el adulto tiene que actuar como referente frente al menor de edad. También añade el hecho de compartir Internet, definiendo este concepto como el diálogo familiar y la revisión de las reglas consensuadas dentro de las familias (Melamud et al., 2007). Uno de los riesgos más frecuentes es el hecho de confiar en cada persona con la que hablan en Internet, sea conocida o no, y aportarle datos personales. Eso conlleva que muchos menores, posteriormente, sean victimizados por contactos que conocieron a través de Internet. El hecho de limitar la comunicación en Internet con personas desconocidas disminuirá el riesgo de victimización $y$, para ello, debe de haber una figura paternal que le avise de dicho peligro y supervise que ese tipo de actividades no se realizan (Marcum et al., 2010). Se ha encontrado que una buena comunicación con los padres también reduce la exposición de los menores a riesgos en Internet (González-Ortega et al., 2008).

\section{OBjetivos}

Este estudio tiene como objetivo principal analizar la victimización por ciberacoso de los menores de un instituto público en Jerez de la Frontera y su relación con factores de riesgo y factores protectores. Para lograr este objetivo, se han definido tres sub-objetivos. El primer sub-objetivo es determinar si los estudiantes de ese instituto de Jerez de la Frontera son víctimas de ciberacoso. El segundo sub-objetivo es determinar si los estudiantes del instituto encuestado de Jerez de la Frontera llevan a cabo conductas de riesgos en Internet y si cuentan con factores de protección. Por último, el tercer objetivo de investigación es determinar si la victimización por ciberacoso está relacionada con las conductas de riesgo en Internet y los factores de protección.

\section{Metodología}

\section{A. Muestra}

En este estudio, exploratorio inicial, participaron 137 estudiantes de un instituto público de la ciudad de Jerez de la Frontera (Cádiz). La muestra está compuesta por 67 alumnos $(48,9 \%)$ y 70 alumnas $(51,1 \%)$, con edades comprendidas entre los 11 y los 17 años $(M=14,28 ; D E$ $=1,68)$, provenientes de los cursos de $1^{\circ} \mathrm{ESO}$ a $1^{\circ}$ de 
Ramírez, A. M. (2020). Factores de riesgo y de protección y victimización por ciberacoso entre menores en Jerez de la Frontera: un estudio exploratorio. Behavior \& Law Journal, 6(1), 51-63.

Bachillerato. El tipo de muestreo escogido inicialmente fue por conglomerados, que habría permitido obtener participantes según tres niveles socioeconómicos colegios de zonas de nivel alto, medio y bajo- para comparar los resultados entre ellos. Sin embargo, solo un instituto, clasificado de nivel socioeconómico medio, pudo participar en este estudio debido al inicio del Estado de Alarma causado por la crisis sanitaria del Covid-19, por lo que finalmente llevamos a cabo un muestreo incidental. En la Figura 1 puede encontrarse la distribución de los participantes por edad y sexo.

Figura 1. Distribución de la muestra por sexo y edad $(N=137)$.

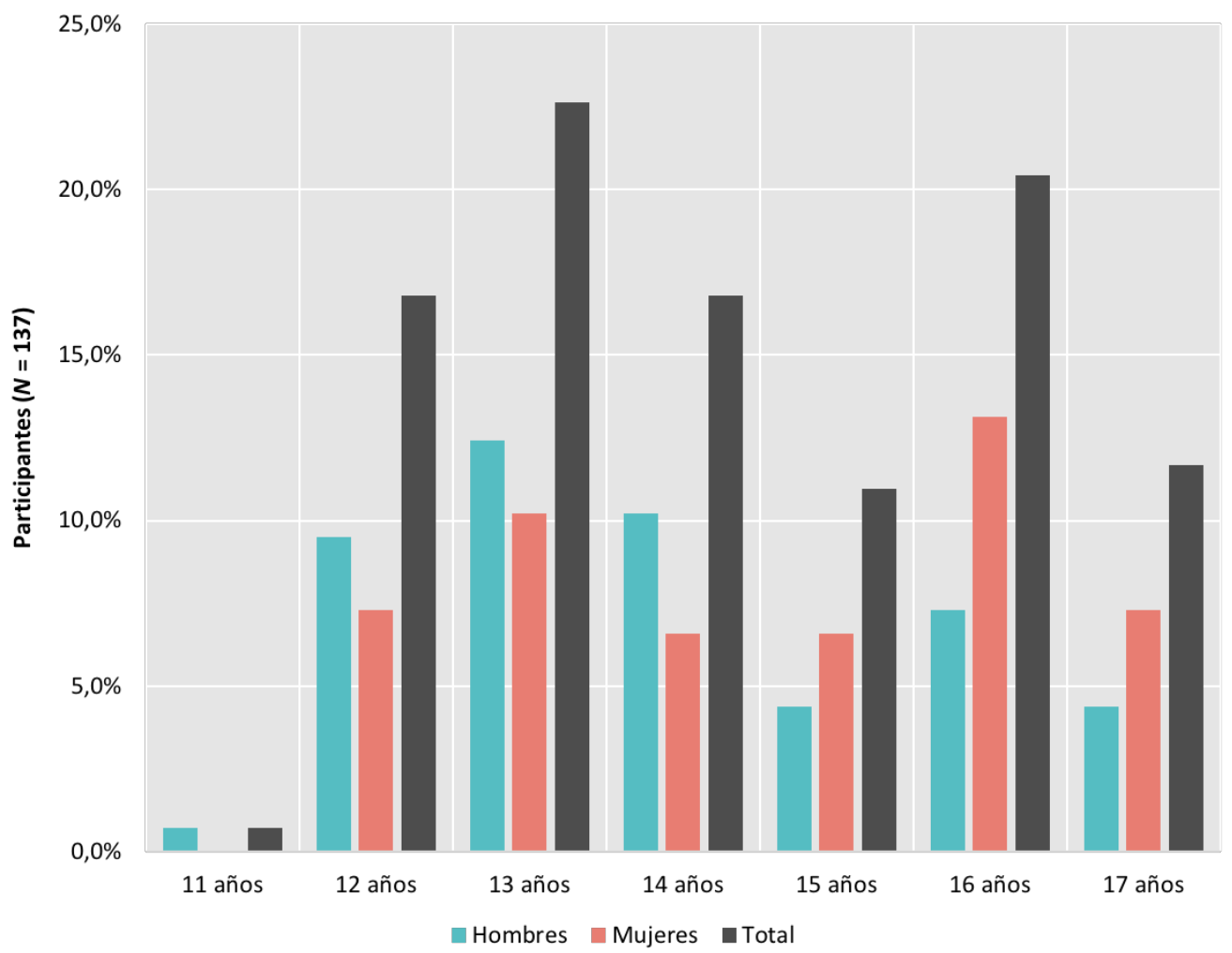

\section{B. Cuestionario}

El cuestionario se compone de cuatro grupos de preguntas: (1) preguntas sociodemográficas donde los participantes indicaron su edad, sexo y curso en el que se encontraban; (2) preguntas relacionadas con el uso de Internet, incluyendo las horas que pasan conectados, dónde se conectan y qué tipo de redes sociales tienen, así como los riesgos a los que pueden estar expuestos; (3) pregunta sobre victimización online; y (4) preguntas para medir la prevención y apoyo que reciben los menores de edad. En el Anexo 1 se puede consultar el cuestionario implementado. A continuación, se explican los tipos de variables de este estudio.

La variable dependiente del estudio -pregunta nueve del cuestionario- midió si el participante había sufrido alguna de las conductas de acoso que se daban como opciones. Para formular las opciones, se siguieron las conductas de ciberacoso definidas por Bartrina Andrés (2014), quien diferencia entre el envío de mensajes desagradables (flaming), envío de imágenes o videos con contenido desagradable (harassment), envío de imágenes o información con tono despectivo (denigration) o amenazas de daño o intimidación (cyberstalking). Además, para diseñar esta pregunta nos basamos en el estudio de Garaigordobil y Aliri (2013), quienes encuentran que las mujeres sufren más de cinco conductas relacionadas con el ciberacoso y que este es mayor que en hombres.

Dos tipos de variables independientes se incluyeron en el análisis. Por un lado, las variables para medir conductas de riesgos en Internet - preguntas de la uno a la ocho del cuestionario-. En estas preguntas se analizaron el tiempo, el lugar dónde utilizaban Internet y las redes sociales que los estudiantes tienen, así como el contenido de estas. También se recogió la realización de conductas categorizadas como "de bajo riesgo" (i.e., jugar a juegos 
Ramírez, A. M. (2020). Factores de riesgo y de protección y victimización por ciberacoso entre menores en Jerez de la Frontera: un estudio exploratorio. Behavior \& Law Journal, 6(1), 51-63.

online, subir fotos y vídeos a mis redes, y utilizar webcam o vídeo llamada), y la realización de conductas categorizadas como "de riesgo alto" (i.e., he añadido a mi lista de amigos a personas que nunca he conocido, he buscado nuevos amigos en Internet, he fingido ser una persona que no era, dando nombres falsos, y he hablado con desconocidos). Por otro lado, en las variables para medir los factores protectores en Internet - pregunta 10 y 11 del cuestionario- se incluyen la conducta de contactar con otras personas cuando ven algo que no les gusta en Internet y los consejos recibidos por parte de familiares, profesores, policía, etc. $(0=$ no; $1=$ sî).

\section{Recopilación de datos y análisis}

En un principio incluimos en el diseño de la investigación el objetivo de recoger datos según diferentes niveles socioeconómicos, con el fin de comprobar si dicho nivel influía en los riesgos, para poder constatar lo que afirmó el sociólogo Tezanos (2006). Sin embargo, como se ha señalado arriba, el inicio del Estado de Alarma a raíz de la crisis sanitaria producida por el Covid-19 y el subsiguiente cierre de todas las instituciones educativas impidió que se pudieran seguir recogiendo los datos.

Los cuestionarios fueron implementados en las clases seleccionadas por la Dirección del Instituto. Todas las preguntas del cuestionario fueron leídas en voz alta antes de que el alumnado contestara para asegurar su adecuada comprensión. Una vez recopilados los cuestionarios, los resultados fueron introducidos manualmente en una base de datos. Posteriormente, se realizaron análisis descriptivos, bivariados y de regresión para responder a los objetivos de la investigación.

\section{Consideraciones éticas}

Siguiendo las recomendaciones de Díaz Fernández (2019) sobre cómo investigar con temas sensibles y con el objetivo de preservar los derechos de los menores, los cuestionarios no recopilaban el nombre del participante. Además, se elaboró una hoja informativa para el AMPA y otra para la Dirección del Instituto explicando los objetivos de la investigación. Tras su aprobación y disposición a colaborar, se implementaron los cuestionarios con los alumnos. Además, la encuesta no recopila ningún tipo de información sensible que pudiera identificar al alumno. La participación de los alumnos fue voluntaria, no se proporcionó ningún tipo de incentivo.

\section{Resultados}

El primer objetivo de esta investigación era determinar si los estudiantes de institutos de Jerez de la Frontera son víctimas de ciberacoso, aunque, debido a las limitaciones del trabajo de campo, solo pudimos comprobar este objetivo en el instituto de estudio. En total, obtuvimos que el 44,5\% $(n=61)$ de los estudiantes encuestados habían sido víctimas de algún tipo de ciberacoso. En la Figura 2 se pueden consultar los tipos de cibervictimización sufridos por los participantes.

Figura 2. Porcentaje de participantes por tipos de cibervictimización $(N=137)$.

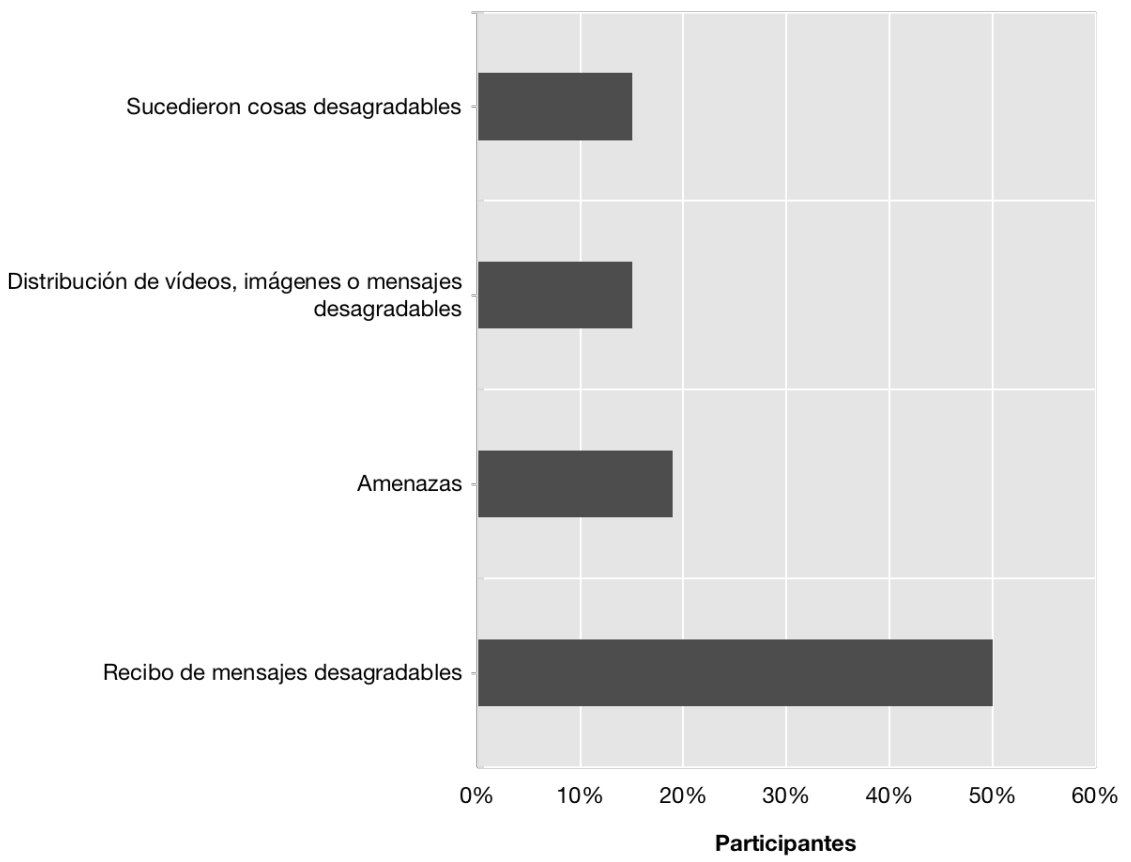


Ramírez, A. M. (2020). Factores de riesgo y de protección y victimización por ciberacoso entre menores en Jerez de la Frontera: un estudio exploratorio. Behavior \& Law Journal, 6(1), 51-63.

Además, obtuvimos que la red social más popular entre los menores encuestados es Instagram, seguida de Twitter (Figura 3), siendo el 81,8\% $(n=112)$ los que tienen perfiles privado frente al $12,4 \%(n=17)$ que lo tienen público.

Figura 3. Porcentaje de participantes por tipo de red social donde tienen perfil personal.

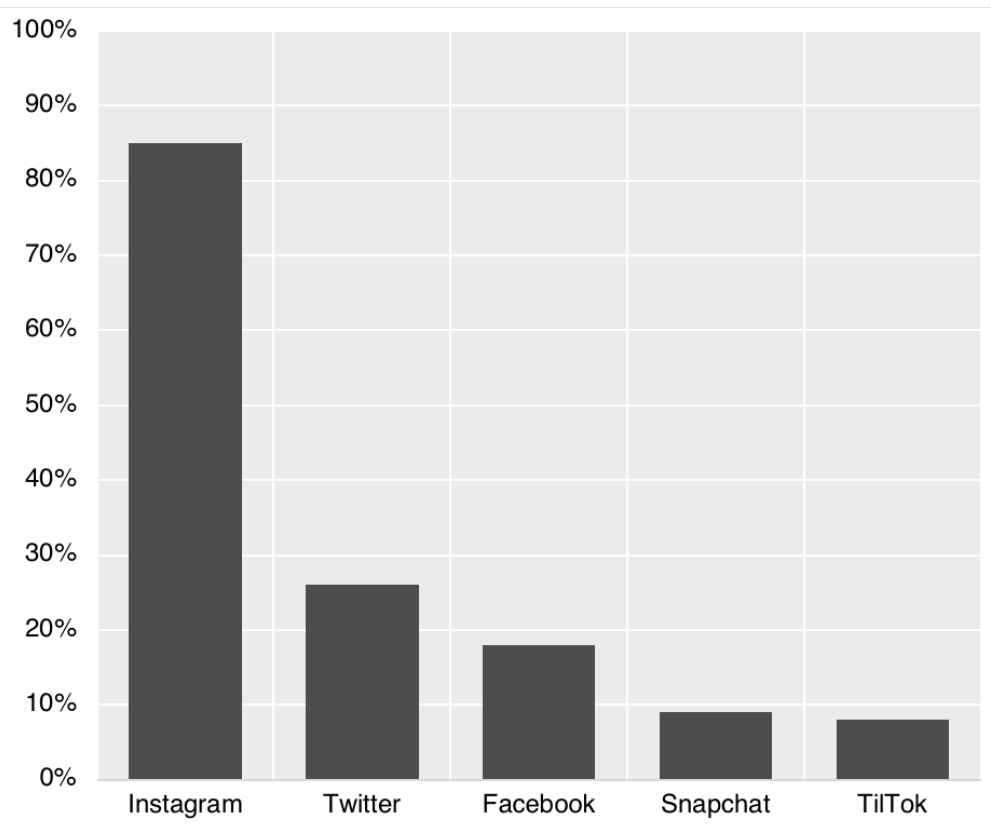

El segundo objetivo era determinar si los estudiantes del instituto encuestado de Jerez de la Frontera llevan a cabo conductas de riesgos en Internet y si cuentan con factores de protección. Obtuvimos que el 70,8\% $(n=97)$ incluye información personal en sus redes sociales y el $64,2 \%(n=88)$ de los estudiantes habla con desconocidos a través de Internet. Por último, obtuvimos que los menores añaden como amigos a perfiles que nunca ha conocido en persona $(51,8 \%, n=71)$, buscan nuevos amigos en Internet $(21,2 \%, n=29)$, fingen ser una persona a la que se es por Internet $(15,3 \%, n=21)$, y envían una foto propia a alguien que nunca han visto $(7,3 \%, n=10)$.
Por su parte, solo el 10,9\% $(n=15)$ no se comunica con nadie cuando le sucede algo desagradable en Internet., el 39,4\% $(n=54)$ se comunican con sus madres o padres, el 22,6\% (n=31) lo hacen con sus hermanos/as, el $81 \%$ $(n=111)$ con sus amigos, el $22,6 \%(n=31)$ con algún compañero de clase, un 2,2\% $(n=3)$ con sus profesores, el $11,7 \%(n=16)$ con otras personas. Por consiguiente, el $91,9 \%(\mathrm{n}=126)$ recibió algún consejo de cómo usar Internet de forma segura (Figura 4). Por tanto, podemos observar que la mayoría de los estudiantes reciben consejos por parte de sus padres o madres, es decir, consejos derivados del ámbito familiar.

Figura 4. Porcentaje de participantes según los consejos recibidos.

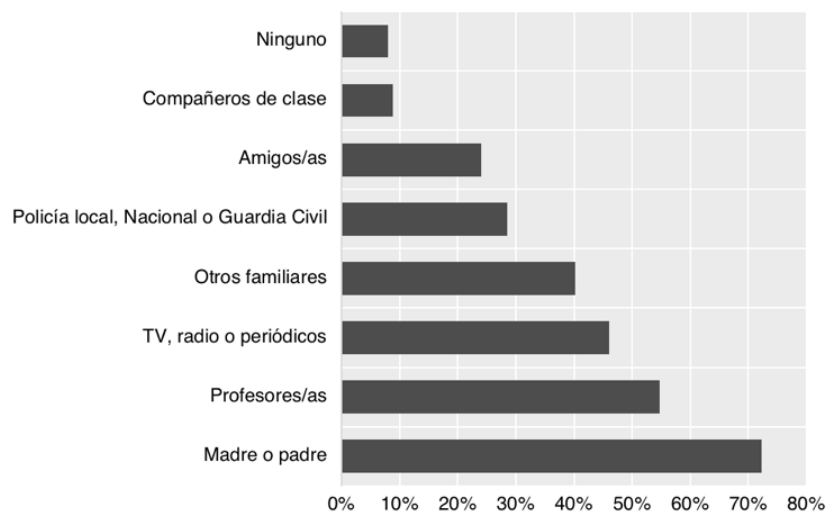


Ramírez, A. M. (2020). Factores de riesgo y de protección y victimización por ciberacoso entre menores en Jerez de la Frontera: un estudio exploratorio. Behavior \& Law Journal, 6(1), 51-63.

El tercer objetivo de nuestra investigación es determinar si la victimización por ciberacoso está relacionada con factores de riesgo y de protección. Encontramos correlaciones estadísticamente significativas entre la victimización por ciberacoso y las horas que utilizan Internet $\left(X^{2}=0,217 p<0,05\right)$, así como el número de redes sociales en las que tienen un perfil propio $\left(X^{2}=0,211 ; p<0,05\right)$, con el número de riesgos bajos $\left(X^{2}=0,202 ; p<0,05\right)$ y el número de riesgos altos $\left(X^{2}=0,349 ; p<0,001\right)$. Según estos resultados, la única variable que no se relaciona significativamente con la victimización es la cantidad de información personal que hayan incluido en sus redes sociales (ver Tabla 1). Sin embargo, la correlación no nos permite saber la dirección de la influencia de las variables independientes sobre la victimización por ciberacoso. Por ello, para conocer cuál es la influencia de las variables independientes sobre la variable dependiente, se han llevado a cabo dos análisis de regresión (logística y lineal).

Tabla 1. Matriz de correlaciones entre variables la variable dependiente (13), las independientes y las sociodemográficas

\begin{tabular}{|c|c|c|c|c|c|c|c|c|c|c|c|c|c|c|}
\hline & 1 & 2 & 3 & 4 & 5 & 6 & 7 & 8 & 9 & 10 & 11 & 12 & 13 & 14 \\
\hline 1. Sexo & - & & & & & & & & & & & & & \\
\hline 2. Edad &, $175^{*}$ & - & & & & & & & & & & & & \\
\hline 3. Curso &, $181^{*}$ &, $947^{* *}$ & - & & & & & & & & & & & \\
\hline 4. Móvil propio & ,076 &, $270^{* *}$ &, $268^{* *}$ & - & & & & & & & & & & \\
\hline $\begin{array}{l}\text { 5. Horas al día en } \\
\text { Internet }\end{array}$ & ,022 &, $453^{* *}$ &, $421^{* *}$ &, $191^{*}$ & - & & & & & & & & & \\
\hline 6. Redes Sociales & ,141 &, $327^{* *}$ &, $329^{* *}$ &, $199^{*}$ &, $377^{* *}$ & - & & & & & & & & \\
\hline $\begin{array}{l}\text { 7. Privacidad del } \\
\text { perfil }\end{array}$ &, $228^{* *}$ &,- 047 &,- 053 &,- 070 &,- 060 &,- 060 & - & & & & & & & \\
\hline $\begin{array}{l}\text { 8. Tipo de foto } \\
\text { de perfil }\end{array}$ &, $234^{* *}$ &, $493^{* *}$ &, $454^{* *}$ & ,166 &, $266^{* *}$ &, $422^{* *}$ &, 000 & - & & & & & & \\
\hline $\begin{array}{l}\text { 9. Información } \\
\text { personal }\end{array}$ & 022 &, $200^{*}$ &, $199^{*}$ &, 066 &, $180^{*}$ &, $270^{* *}$ &,$- 210^{*}$ &, $189^{*-}$ & - & & & & & \\
\hline $\begin{array}{l}\text { 10. Habla con } \\
\text { desconocidos en } \\
\text { Internet }\end{array}$ &,- 029 &, $373^{* *}$ &, $371^{* *}$ &, $212^{*}$ &, $237^{* *}$ &, $228^{* *}$ &,- 081 &, $299^{* *}$ &, $385^{* *}$ & - & & & & \\
\hline $\begin{array}{l}\text { 11. Cantidad de } \\
\text { riesgos bajos }\end{array}$ &,- 146 & ,123 &, 112 & ,022 &, $271^{* *}$ &, $240^{* *}$ & ,001 &, $232^{* *}$ &, $313^{* *}$ &, $246^{* *}$ & - & & & \\
\hline $\begin{array}{l}\text { 12. Cantidad de } \\
\text { riesgo alto }\end{array}$ &, 015 &, $299^{* *}$ &, $318^{* *}$ &, 055 &, $314^{* *}$ &, $282^{* *}$ &,- 146 &, $235^{* *}$ &, $197^{*}$ &, $279^{* *}$ &, $324^{* *}$ & - & & \\
\hline $\begin{array}{l}\text { 13. Victimización } \\
\text { por ciberacoso }\end{array}$ &, 120 & 053 &, 058 &, 030 &, $217^{*}$ &, $211^{*}$ & ,136 & , 166 & 074 & ,130 & ,202* &, $349^{* *}$ & - & \\
\hline 14. Confianza & 056 &, $335^{* *}$ &, $375^{* *}$ &, $281^{* *}$ &, $249^{* *}$ &, $432^{* *}$ &,- 027 &, $387^{* *}$ &, $177^{*}$ &, $301^{* *}$ &, 148 &, $280^{* *}$ &, $234^{* *}$ & - \\
\hline 15. Consejos & $244^{* *}$ & 071 & ,108 &, $179^{*}$ & ,088 &, 120 &,- 008 &, $213^{*}$ &,- 009 & 059 & 099 &, $284^{* *}$ &, $321^{* *}$ &, $180^{*}$ \\
\hline
\end{tabular}

Nota: Correlaciones significativas a los niveles ${ }^{*} \mathrm{p}$-valor $<0,05,{ }^{* *} \mathrm{p}$-valor $<0,001$.

Para estudiar si tener móvil propio, tener redes sociales, tener una foto completa en las redes sociales y tener perfil publico incrementan la probabilidad de ser víctima por ciberacoso, hemos realizado un análisis de regresión logística. Como se puede observar en la Tabla 2, no se ha encontrado relación entre la victimización por ciberacoso y dichas variables.

Para comprobar el resto de las hipótesis del tercer objetivo se ha llevado a cabo un análisis de regresión lineal. Lo primero que hemos obtenido es el valor numérico de Durbin-Watson, que es de 1,87, por lo que se considera que el modelo es adecuado ya que dicho valor debe oscilar entre 1,5 y 2,5 para que se considere que los datos siguen una distribución normal y que, por tanto, podamos realizar la regresión lineal. En la Tabla 3 se resumen los resultados del análisis de regresión. 
Ramírez, A. M. (2020). Factores de riesgo y de protección y victimización por ciberacoso entre menores en Jerez de la Frontera: un estudio exploratorio. Behavior \& Law Journal, 6(1), 51-63.

Tabla 2 . Regresión logística para la victimización por ciberacoso

\begin{tabular}{|c|c|c|c|}
\hline$\overline{\text { Variable }}$ & $\bar{B}$ & Error & $\overline{\operatorname{Exp}(b)}$ \\
\hline Constante & $-21,976$ & 23205,4 & 0,00 \\
\hline Móvil propio & $-0,10$ & 1,03 & 0,89 \\
\hline $\begin{array}{l}\text { Perfil propio en } \\
\text { RRSS }\end{array}$ & 20,81 & 23205,4 & 0,00 \\
\hline $\begin{array}{l}\text { Tipo de foto en el } \\
\text { perfil }\end{array}$ & 0,28 & 0,229 & 1,325 \\
\hline $\begin{array}{l}\text { Privacidad del } \\
\text { perfil }\end{array}$ & 0,88 & 0,570 & 2,413 \\
\hline $\mathrm{R}^{2}$ de Cox y Snell & & & 0,061 \\
\hline
\end{tabular}

Obtenemos que la victimización por ciberacoso está relacionada con los estudiantes más jóvenes, así como con aquellos que llevan a cabo actividades de riesgos altos y con las personas que contacta cuando ve algo que no le gusta en Internet. Sin embargo, obtenemos que no influye en la victimización las horas, las redes sociales, la información personal, los riesgos bajos ni los consejos recibidos. Encontramos que las conductas de riesgos considerados como bajos no incrementan ni disminuyen la probabilidad de ser víctima por ciberacoso. También encontramos que la cantidad de personas con la que contactan los menores cuando ven algo que no les gusta en Internet se relaciona positivamente con la probabilidad de ser víctima de ciberacoso. Sin embargo, obtenemos que la relación entre los consejos recibidos y ser víctima de ciberacoso no es significativa. Obtenemos que las variables de nuestro modelo explican un $20,4 \%$ de la varianza de la victimización por ciberacoso. Por último, obtuvimos que los estudiantes varones y las mujeres sufren de media el mismo número de victimizaciones, $t(135)=-1,64, p$-valor $=0,104$.

Tabla 3 . Regresión lineal para la victimización por ciberacoso

\begin{tabular}{|c|c|c|c|c|}
\hline Variable & $B$ & Error & $t$ & $p$-valor \\
\hline Constante & 0,885 & 0,677 & 1,31 & 0,193 \\
\hline Edad & $\overline{0}, 117$ & 0,052 & $-2,26$ & 0,026 \\
\hline Horas & 0,124 & 0,103 & 1,19 & 0,234 \\
\hline Redes sociales & 0,086 & 0,089 & 0,97 & 0,336 \\
\hline Información personal & 0,016 & 0,072 & 0,23 & 0,820 \\
\hline Riesgos bajos & 0,049 & 0,109 & 0,45 & 0,651 \\
\hline Riesgos altos & 0,261 & 0,105 & 2,48 & 0,014 \\
\hline $\begin{array}{l}\text { Personas con las que habla en } \\
\text { Internet }\end{array}$ & 0,193 & 0,087 & 2,23 & 0,028 \\
\hline Consejos recibidos & 0,102 & 0,055 & 1,84 & 0,068 \\
\hline $\mathrm{R}^{2}$ & & & & 0,251 \\
\hline $\mathrm{R}^{2}$ ajustado & & & & 0,204 \\
\hline $\mathrm{N}^{\circ}$ observaciones & & & & 137 \\
\hline
\end{tabular}

Nota: $B=$ coeficiente no estandarizado, error $=$ error estándar. Variables significativas a nivel $p$-valor $<0,05$ resaltadas en negrita.

\section{DISCUSIÓN E IMPLICACIONES PARA LA PREVENCIÓN}

La tecnología avanza sin cesar y la mayoría de la población tiene acceso a Internet, como afirma el INE en 2018, reiterándolo en 2019. A este dato, le sumamos el anonimato y globalización que muestra el ciberespacio, junto a su peligrosidad (Maimon \& Louderback, 2019). En consecuencia, vemos cómo el cibercrimen, en general, está aumentando (p.ej., Kemp et al., 2020; Miró-Llinares \& Moneva, 2019), y en concreto, el ciberacoso (Cosma et al., 2020). Los menores de edad son la víctima perfecta para los delincuentes debido a su inocencia y a la poca información que tienen sobre cómo usar Internet de forma segura (González-Ortega et al., 2008). No son conscientes de los peligros a los que se enfrentan y confían en la seguridad del uso de Internet, según el INE (2019). El objetivo general del estudio era analizar las conductas de riesgo, factores protectores y victimización por ciberacoso entre menores en Jerez de la Frontera. Para abordar este objetivo se ha realizado una encuesta a los alumnos de un instituto de Jerez de la Frontera, para poder estudiar si los menores de edad, de 11 a 17 años, tienen probabilidad de ser víctimas de ciberacoso, analizando las conductas de riesgos y los factores de protección.

El análisis de los datos demostró que las variables que hemos considerado factores de riesgos aumentan la probabilidad de ser víctima de ciberacoso, específicamente las horas que pasan en Internet, el uso de las redes sociales y los riesgos consideramos como bajos y altos, en línea con otros estudios similares sobre ciberacoso entre menores (Bossler et al., 2012; Hinduja \& Patchin, 2008; Marcum et al., 2010; Mishna et al., 2012). El porcentaje de victimización entre los alumnos encuestados es del 44,5\%, una prevalencia muy alta comparada con la obtenida por otros estudios (Selkie et al., 2016). Sin embargo, cuentan con factores de protección, siendo la información que reciben de cómo usar Internet de forma segura proporcionada por los padres y profesores. La mayoría de ellos acuden a alguien cuando ven que corren algún tipo de riesgo, sobre todo a sus amigos.

Además, tenemos que valorar el hecho de que los menores carecen de recursos para responder frente a situaciones desagradables, sin saber cómo actuar ni a quién acudir porque no son conscientes de la gran importancia de los factores de protección (Marcum et al., 2010). Por ejemplo, en el caso de los colegios, los menores son conscientes de que los centros educativos tienen la 
Ramírez, A. M. (2020). Factores de riesgo y de protección y victimización por ciberacoso entre menores en Jerez de la Frontera: un estudio exploratorio. Behavior \& Law Journal, 6(1), 51-63.

responsabilidad a la hora de gestionar conflictos en los que están implicados su alumnado, pero estos parecen no tener la suficiente confianza en ellos. Con esto queremos resaltar a la importancia de la sensibilización y la concienciación sobre los riesgos, así como herramientas y recursos necesarios para afrontarlos, ya que es la clave a la reducción de los ciberdelitos y la clave de la autoprotección. Además de la concienciación a menores, se debería de incidir en la educación a los padres y profesores/as, ya que se ha demostrado en repetidos estudios que los programas que de prevención del ciberacoso que incluyen educación a los padres tienen resultados positivos en la reducción del ciberacoso y la cibervictimización (Cross et al., 2016; Del Rey et al., 2012, 2016; Garaigordobil \& Martínez-Valderrey, 2016; Gradinger et al., 2016; Ortega-Ruiz et al., 2012; Salmivalli et al., 2011; Schultze-Krumbholz et al., 2016; Williford et al., 2013; Wölfer et al., 2014).

Dadas las limitaciones de validez externa del estudio, debemos señalar que se ha realizado un análisis de carácter exploratorio sobre el uso de las redes rociales y los factores asociados a la cibervictimización, lo que nos permite realizar algunas recomendaciones que, no obstante, deben ser tomadas con cautela. En primer lugar, recomendamos realizar programas de educación y concienciación a los padres, madres y profesorado, a fin de que estos sepan identificar las situaciones en las que se está produciendo el ciberacoso y cómo reaccionar de forma adecuada. Además, se debe promover que los menores tengan confianza tanto en sus progenitores como en el profesorado, con el fin de que les soliciten ayuda en el caso de ser víctimas de ciberacoso en Internet. Esto no significaría que tanto padres como profesores sean los únicos que deben intervenir en la prevención. También se propone el trabajo activo de los criminólogos/as y otros profesionales especializados en los colegios e institutos de para dar pautas más específicas sobre cómo abordar este tema, en línea con las recomendaciones realizadas sobre las funciones que puede asumir la criminología en la lucha contra el acoso escolar (Del Real, 2015). También proponemos que los alumnos de cursos superiores preparen una sesión para hacerles llegar esta información a los alumnos menores, en una suerte de programa de mentoría. De esta forma, habría una autoenseñanza de los mayores y una información recibida por sus iguales desde el punto de vista de los menores (García et al., 2010). Esta propuesta se fundamenta en que dentro del grupo de iguales alumnos de un instituto- la confianza es mayor y es más probable que los menores interpreten los consejos como algo beneficioso para ellos.

\section{CONCLUSIONES Y LIMITACIONES}

El objetivo de esta investigación es analizar las conductas de riesgo, factores protectores y victimización por ciberacoso entre menores de un instituto público en Jerez de la Frontera, ya que la tecnología avanza interrumpidamente y con ella la victimización en el ciberespacio. Para conseguir nuestros objetivos realizamos una amplia revisión de artículos criminológicos sobre los ciberdelitos y cómo afectan estos a los menores de edad. A partir de esa información se definieron una serie de preguntas para elaborar la encuesta que, posteriormente, se implementó en 137 alumnos de un instituto de Jerez de la Frontera. Cada una de las preguntas estaba compuesta por una serie de variables, entre las que se incluían conductas de riesgos -por ejemplo, las horas que pasa conectado a Internet, los perfiles de redes sociales que posee o si habla con desconocidos en el ciberespacio-y los factores de protección -la información que reciben sobre cómo usar Internet de forma segura o con quien interactúa cuando ve algo en Internet que no le agrada-.

En general, nuestros resultados muestran la relación entre las conductas de riesgos y los factores de protección con la victimización en el ciberespacio entre los alumnos encuestados. Además, se ha encontrado algunas variables claves que explican la victimización por ciberacoso, estas son la edad (estudiantes más jóvenes corren mayor riesgo de victimización), los que llevan a cabo conductas de riesgo considerados "altos", el número de redes sociales que poseen y el número de personas con las que contacta cuando ven algo que no le gusta en Internet, clasificada, esta última, como factor de protección.

Este estudio podría servir como base para desarrollar un trabajo de campo más extenso con el fin de intervenir en la reducción de las conductas de riesgos y evaluar el progreso y los resultados. Sin embargo, este estudio tiene algunas limitaciones que son necesarias señalar. En primer lugar, el tamaño de la muestra, que no es representativo y es relativamente pequeño -137 alumnos-, y obtenida en solo un instituto de Jerez de la Frontera -Cádiz-. Por tanto, los resultados no son generalizables. Tampoco se ha podido diferenciar entre los diferentes niveles socioeconómicos como se preveía realizar en un principio. Además, no se ha podido tener en cuenta la opinión del profesorado ni de los padres, que sería interesante para estudiar los factores de protección en profundidad.

Por consiguiente, las futuras investigaciones deberán 
Ramírez, A. M. (2020). Factores de riesgo y de protección y victimización por ciberacoso entre menores en Jerez de la Frontera: un estudio exploratorio. Behavior \& Law Journal, 6(1), 51-63.

analizar esta cuestión utilizando una muestra más amplia, representativa del universo de estudio donde, en un primer lugar, se midan las conductas de riesgos y los factores de protección. En segundo lugar, se establezca un protocolo de actuación y concienciación tanto para los alumnos como para los educadores - profesorado y padres/madres-. Realizar una encuesta a los padres y madres, así como a los profesores para poder concluir el conocimiento que tienen ellos sobre la probabilidad que tienen los menores de sufrir victimización por ciberacoso. Este protocolo y plan de prevención se evaluaría mediante un análisis de la evolución de las conductas de riesgo y los factores de protección y su relación con la probabilidad de victimización por ciberacoso del menor de edad. Además, se deberían clasificar en los diferentes niveles socioeconómicos -bajo, medio, alto- para analizar si hay alguna diferencia entre los institutos de Jerez de la Frontera. En general, se deben incluir nuevas variables de estudio. Por último, proponemos una investigación evaluativa de tipo experimental en la que se analizaría la probabilidad que tienen los alumnos de sufrir victimización por ciberacoso antes y después de llevar a cabo una serie de medidas preventivas, tanto con ellos como con sus educadores -padres y profesorado-.

\section{AGRADECIMIENTOS}

A María del Mar Martín Aragón y Cristina del Real Castrillo, de la Universidad de Cádiz, por sus comentarios a versiones previas de este artículo.

\section{REFERENCIAS}

Andrés, M. J. B. (2014). Conductas de ciberacoso en niños y adolescentes. Hay una salida con la educación y la conciencia social. Educar, 50(2), 383-400.

Aponte Rueda, D. R., Castillo Chávez, P., González Estrella, J. E., Aponte Rueda, D. R., Castillo Chávez, P., \& González Estrella, J. E. (2017). Prevalencia de adicción a internet y su relación con disfunción familiar en adolescentes. Revista Clínica de Medicina de Familia, 10(3), 179-186.

Bossler, A. M., Holt, T. J., \& May, D. C. (2012). Predicting Online Harassment Victimization Among a Juvenile Population. Youth \& Society, 44(4), 500-523. https://doi.org/10.1177/0044118X11407525

Catalina García, B., López de Ayala López, M., \& García Jiménez, A. (2014). Los riesgos de los adolescentes en Internet: Los menores como actores y víctimas de los peligros de Internet. Revista Latina de Comunicación Social, 69, 462-485. https://doi.org/10.4185/RLCS-2014-1020

Cosma, A., Walsh, S. D., Chester, K. L., Callaghan, M., Molcho, M., Craig, W., \& Pickett, W. (2020). Bullying victimization: time trends and the overlap between traditional and cyberbullying across countries in Europe and North America. International Journal of Public Health, 65, 75-85. https://doi.org/10.1007/s00038-019-01320-2

Cross, D., Shaw, T., Hadwen, K., Cardoso, P., Slee, P., Roberts, C., Thomas, L., \& Barnes, A. (2016). Longitudinal impact of the Cyber Friendly Schools program on adolescents' cyberbullying behavior. Aggressive Behavior, 42(2), 166-180. DOI: $10.1002 / \mathrm{ab} .21609$

Cruzado Díaz, L., Matos Retamozo, L., \& Kendall Folmer, R. (2012). Adicción a internet: Perfil clínico y epidemiológico de pacientes hospitalizados en un instituto nacional de salud mental. Revista Medica Herediana, 17(4), 196. https://doi.org/10.20453/rmh.v17i4.879

Del Real, C. (2015). Criminólogos: instrucciones de uso. Un análisis de futuras lineas aplicadas en criminología. [Trabajo de Fin de Grado, Universidad de Sevilla]. idUS. http://hdl.handle.net/11441/30120

Del Rey, R., Casas, J., \& Ortega, R. (2012). The ConRed Program, an evidence-based practice. Comunicar, 20(39), 129-138. https://doi.org/10.3916/C39-2012-03-03

Del Rey, R., Casas, J. A., \& Ortega, R. (2016). Impact of the ConRed program on different cyberbulling roles. Aggressive Behavior, 42(2), 123-135. https://doi.org/10.1002/ab.21608

Díaz Fernández, Antonio M. (2019). La investigación de temas sensibles en Criminología y Seguridad. Madrid: Tecnos.

Echeburúa, E., \& de Corral, P. (2010). Adicción a las nuevas tecnologías y a las redes sociales en jóvenes: Un nuevo reto. Adicciones, 22(2), 91-96. https://doi.org/10.20882/adicciones.196

Gamito, R., Aristizabal, P., Vizcarra, M. T., \& Olosolo, M. (2017). La necesidad de trabajar los riesgos de Internet en el aula. Profesorado. Revista de currículum y formación de profesorado, 21(3), 409-426.

Garaigordobil, M., \& Aliri, J. (2013). Cyberbullying in the Basque Country: Sex differences in victims, perpetrators and observers. Behavioral Psychology, 21(3), 461-474.

García, M. J. G., López, M. C. G., \& Quintana, P. J. V. (2010). Mentoría entre iguales: Alumnos que comparten experiencias y aprendizaje. XVI Jornadas de Enseñanza Universitaria de la Informática, 8.

García Oliva, C. (2017). Uso problemático de Internet en adolescentes y factores asociados [Tesis Doctoral, Universidad Miguel Hernández de Elche]. https://pdfs.semanticscholar.org/c10a/9e20a75b0ecc1df6 c553440d13c8981f885c.pdf

Garmendia Larrañaga, M., Jiménez Iglesias, E., Casado, M. Á., \& Mascheroni, G. (2016). Riesgos y oportunidades en internet y uso de dispositivos móviles entre menores españoles (2010-2015) (Informe de proyecto N. ${ }^{\circ}$ CSO2013-47304-R; Not Children Go Mobile, p. 112). Red.es/Universidad del País Vasco. 
Ramírez, A. M. (2020). Factores de riesgo y de protección y victimización por ciberacoso entre menores en Jerez de la Frontera: un estudio exploratorio. Behavior \& Law Journal, 6(1), 51-63.

https://addi.ehu.es/bitstream/handle/10810/21546/Infor me $\% 20$ NCGM $\% 20$ Espa $\%$ C3 $\%$ B1a $\% 202010-$

2015.pdf?sequence $=1$

Garmendia, M., Martínez, G., Casado, M. Á., \& Garitaonandia, C. (2012). Los menores en internet. Usos y seguridad desde una perspectiva europea. Quaderns del CAC, XV(1), 37-44.

González-Ortega, I., Echeburúa, E., \& de Corral, P. (2008). Variables significativas en las relaciones violentas en parejas jóvenes: Una revisión. Behavioral Psychology, 16(2), 207-225.

Gradinger, P., Yanagida, T., Strohmeier, D., \& Spiel, C. (2016). Effectiveness and sustainability of the ViSC Social Competence Program to prevent cyberbullying and cybervictimization: Class and individual level moderators. Aggressive Behavior, 42(2), 181-193. https://doi.org/10.1002/ab.21631

Hinduja, S., \& Patchin, J. W. (2008). Cyberbullying: An Exploratory Analysis of Factors Related to Offending and Victimization. Deviant Behavior, 29(2), 129-156. https://doi.org/10.1080/01639620701457816

Instituto Nacional de Estadística. (2018). España en cifras 2018 (España en cifras) [Informe estadístico]. Instituto Nacional de Estadística https://www.ine.es/prodyser/espa_cifras/2018/2/

Jiménez Iglesias, E., \& Garmendia Larrañaga, M. (2015). Percepción de los y las menores de la mediación parental respecto a los riesgos en internet. Revista Latina de Comunicación Social, 70, 49-68.

Kemp, S., Miró-Llinares, F., \& Moneva, A. (2020). The Dark Figure and the Cyber Fraud Rise in Europe: Evidence from Spain. European Journal on Criminal Policy and Research, 26, 293 312. https://doi.org/10.1007/s10610-020-09439-2

Marcum, C. D., Higgins, G. E., \& Ricketts, M. L. (2010). Potential Factors of Online Victimization of Youth: An Examination of Adolescent Online Behaviors Utilizing Routine Activity Theory. Deviant Behavior, 31(5), 381-410. https://doi.org/10.1080/01639620903004903

Marcum, C. D., Ricketts, M. L., \& Higgins, G. E. (2010). Assessing Sex Experiences of Online Victimization: An Examination of Adolescent Online Behaviors Using Routine Activity Theory. Criminal Justice Review, 35(4), 412437. https://doi.org/10.1177/0734016809360331

Melamud, A., Otero, P., \& Nasanovsky, J., Stechina, D., Goldfarb, G., Svetliza, J., Canosa, D., Matamoros, R., \& Ringuelet, L. (2007). Los niños, sus padres, Internet y los pediatras. Archivos Argentinos de Pediatría, 105(4), 368-371.

Miranda de Larra, R. (2005). Los menores en la Red: Comportamiento y navegación segura (N. ${ }^{\circ}$ 6; Cuadernos Sociedad de la Información, p. 34). Fundación Auna.

Miró-Llinares, F. (2012). El cibercrimen. Fenomenología y criminología de la delincuencia en el ciberespacio (1. ${ }^{\text {a }}$ ed.). Marcial Pons. http://www.marcialpons.es/libros/el-

cibercrimen/9788415664185/

Miró-Llinares, F., \& Moneva, A. (2019). What about cyberspace (and cybercrime alongside it)? A reply to Farrell and Birks "Did cybercrime cause the crime drop?". Crime Science, 8(12), 1-5. https://doi.org/10.1186/s40163-019-0107-y

Mishna, F., Khoury-Kassabri, M., Gadalla, T., \& Daciuk, J. (2012). Risk factors for involvement in cyber bullying: Victims, bullies and bully-victims. Children and Youth Services Review, 34(1), 63-70. https://doi.org/10.1016/j.childyouth.2011.08.032

Moneva, A., Miró-Llinares, F., \& Hart, T. C. (2020). Hunter or Prey? Exploring the Situational Profiles that Define Repeated Online Harassment Victims and Offenders. Deviant Behavior, 1-16. https://doi.org/10.1080/01639625.2020.1746135

Navarro, J. N., \& Jasinski, J. L. (2013). Why Girls? Using Routine Activities Theory to Predict Cyberbullying Experiences Between Girls and Boys. Women \&o Criminal Justice, 23(4), 286-303.

Olivia Delgado, A. (2012). Desarrollo cerebral y asunción de riesgos durante la adolescencia. Apuntes de Psicología, 30(3), 477-486.

Ortega-Ruiz, R., Del Rey, R., \& Casas, J. (2012). Knowing, building and living together on Internet and social networks: The ConRed Cyberbullying Prevention Program. International Journal of Conflict and Violence, 6(2), 303-313.

Ramos-Soler, I., López-Sánchez, C., \& Torrecillas-Lacave, T. (2018). Online risk perception in young people and its effects on digital behaviour. Comunicar, 26(56), 71-79. https://doi.org/10.3916/C56-2018-07

Salmivalli, C., Karna, A., \& Poskiparta, E. (2011). Counteracting bullying in Finland: The KiVa program and its effects on different forms of being bullied. International Journal of Behavioral Development, 35(5), 405-411.

Schultze-Krumbholz, A., Schultze, M., Zagorscak, P., Wölfer, R., \& Scheithauer, H. (2016). Feeling cybervictims' pain-The effect of empathy training on cyberbullying. Aggressive Behavior, 42(2), 147-156. https:/ / doi.org/10.1002/ab.21613

Selkie, E. M., Fales, J. L., \& Moreno, M. A. (2016). Cyberbullying Prevalence Among US Middle and High School-Aged Adolescents: A Systematic Review and Quality Assessment. Journal of Adolescent Health, 58(2), 125-133. https://doi.org/10.1016/j.jadohealth.2015.09.026

Williford, A., Elledge, L. C., Boulton, A. J., DePaolis, K. J., Little, T. D., \& Salmivalli, C. (2013). Effects of the KiVa Antibullying Program on cyberbullying and cybervictimization frequency among Finnish youth. Journal of Clinical Child and Adolescent Psychology, 42(6), 820-833.

Wölfer, R., Schultze-Krumbholz, A., Zagorscak, P., Jäkel, A., 
Ramírez, A. M. (2020). Factores de riesgo y de protección y victimización por ciberacoso entre menores en Jerez de la Frontera: un estudio exploratorio. Behavior \& Law Journal, 6(1), 51-63.

Göbel, K., \& Scheithauer, H. (2014). Prevention 2.0:

Targeting cyberbullying@ school. Prevention Science, 15(6), 879-887. https://doi.org/10.1007/s11121-013-0438-y 
Ramírez, A. M. (2020). Factores de riesgo y de protección y victimización por ciberacoso entre menores en Jerez de la Frontera: un estudio exploratorio. Behavior \& Law Journal, 6(1), 51-63.

\section{Anexos.}

Anexo 1. Cuestionario implementado con su correspondiente codificación.

\begin{tabular}{|c|c|c|c|}
\hline $\mathbf{N}^{\mathbf{o}}$ & Preguntas & Posibles respuestas & Codificación \\
\hline \multirow{4}{*}{1} & \multirow{4}{*}{ ¿Cuántas horas al día dedicas a Internet? } & Menos de 1 hora & 1 \\
\hline & & Entre 1 y 2 horas & 2 \\
\hline & & Entre 2 y 3 horas & 3 \\
\hline & & Mas de 3 horas & 4 \\
\hline \multirow{2}{*}{2} & \multirow{2}{*}{ ¿Utilizas Internet en tu móvil propio? } & Sí & 1 \\
\hline & & No & 0 \\
\hline \multirow{4}{*}{3} & \multirow{4}{*}{ ¿Para qué has utilizado Internet estas últimas semanas? } & Buscar información en páginas Web & \multirow{4}{*}{$\begin{array}{c}0=\text { "no" } \\
1=\text { "sí" }\end{array}$} \\
\hline & & Juegos Online & \\
\hline & & Subir fotos o videos a las redes sociales & \\
\hline & & Realizar video llamadas o utilizar la webcam & \\
\hline \multirow{5}{*}{4} & \multirow{5}{*}{ ¿Qué redes sociales tienes? } & Instagram & \multirow{5}{*}{$\begin{array}{c}0=\text { " "no" } \\
1=\text { "sí" }\end{array}$} \\
\hline & & Facebook & \\
\hline & & Twitter & \\
\hline & & Snapchat & \\
\hline & & TikTok & \\
\hline \multirow{2}{*}{5} & \multirow{2}{*}{ ¿Qué tipo de perfil? } & Privado & 1 \\
\hline & & Público & 0 \\
\hline \multirow{7}{*}{6} & \multirow{7}{*}{ ¿Qué elementos está en tu perfil? } & Nombre y/o apellidos reales & \multirow{7}{*}{$\begin{array}{c}0=\text { " no" } \\
1=\text { "sí" }\end{array}$} \\
\hline & & Edad real & \\
\hline & & Dirección & \\
\hline & & Numero de contacto & \\
\hline & & Fotos de solo mi cara & \\
\hline & & Fotos de solo mi cuerpo & \\
\hline & & Fotos de mi cuerpo y mi cara & \\
\hline \multirow{5}{*}{7} & \multirow{5}{*}{ ¿Cuáles de estas cosas has realizado? } & $\begin{array}{l}\text { He añadido a mi lista de amigos a personas que nunca he } \\
\text { conocido }\end{array}$ & \multirow{5}{*}{$\begin{array}{c}0=\text { = "no" } \\
1=\text { ="sí" }\end{array}$} \\
\hline & & He buscado nuevos amigos en Internet & \\
\hline & & He fingido ser una persona que no era, dando datos falsos & \\
\hline & & $\begin{array}{l}\text { He enviado fotos de mi mismo a alguien que nunca he visto } \\
\text { cara a cara }\end{array}$ & \\
\hline & & $\begin{array}{l}\text { He grabado o subido a Internet imágenes o videos de } \\
\text { alguien sin su permiso. }\end{array}$ & \\
\hline \multirow{4}{*}{8} & \multirow{4}{*}{ ¿Alguna vez has estado frente a alguna de estas acciones? } & Me enviaron a través de Internet mensajes desagradables & \multirow{4}{*}{$\begin{array}{c}0=\text { ="no" } \\
1=\text { ="sí" }\end{array}$} \\
\hline & & $\begin{array}{l}\text { Se enviaron videos, imágenes o mensajes desagradables } \\
\text { sobre mi }\end{array}$ & \\
\hline & & Fui amenazado por Internet & \\
\hline & & Sucedieron cosas desagradables & \\
\hline \multirow{7}{*}{9} & & Con mi madre y/o padre & \\
\hline & & Con mi hermano/a & \\
\hline & & Con mi amigo/a & \\
\hline & ¿Con quien contactas cuando ves algo que no te gusta en & Con un compañero de clase & 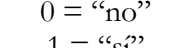 \\
\hline & & Con mi profesor/a & \\
\hline & & Con nadie & \\
\hline & & Con otras personas & \\
\hline & & De mi padre y/o madre & \\
\hline & & De otros familiares & \\
\hline & & De mis profesores/as & \\
\hline & ¿De quien has recibido consejos de cómo usar Internet & De mis amigos/as & $0=$ "no" \\
\hline 10 & de forma segura? & De compañeros de clase & $1=$ "sí" \\
\hline & & De la Policía Local, Nacional o Guardia Civil & \\
\hline & & A través de TV, radio o periódicos & \\
\hline & & No he recibido ningún consejo & \\
\hline & & $\mathrm{Si}$ & 1 \\
\hline 11 & ¿Te gustaría recibir información de este tipo? & No & 0 \\
\hline
\end{tabular}

